\title{
Genetic architecture of differences in oviposition preference between ancestral and derived populations of the seed beetle Acanthoscelides obtectus
}

\author{
N Tucić ${ }^{1,2}$ and D Šešlija ${ }^{2}$ \\ ${ }^{1}$ Faculty of Biology, University of Belgrade, Belgrade, Serbia and ${ }^{2}$ Department of Evolutionary Biology, Institute for Biological Research \\ 'Siniša Stanković', Belgrade, Serbia
}

\begin{abstract}
We investigated the additive, dominance and epistatic genetic effects underlying differentiation in oviposition preference between two populations of the seed beetle Acanthoscelides obtectus evolved in the laboratory for 102 generations on bean and chickpea seeds. We reared and tested females on each of two host legumes. The populations differed in mean oviposition preference; the preference for chickpea was stronger in population reared on the chickpea $(C)$ than in population maintained on common bean $(P)$. Observations in the parental populations indicated that females tend to prefer ovipositioning their eggs on the seeds they have already experienced. The patterns of the means in each of the parental populations and 12 types of
\end{abstract}

hybrids (two $F_{1}$, two $F_{2}$ and eight backcrosses) indicated that population differences in oviposition preference from both rearing hosts could be explained by nonadditive genetic effects. Statistically detectable additive and dominance genetic effects were observed in the most parsimonious model only when females were reared on the chickpea. The most parsimonious models on both rearing hosts suggested a contribution of negative additive $\times$ additive epistasis to the divergence of oviposition preference between the $\mathrm{P}$ and $\mathrm{C}$ populations. This indicates a positive effect of epistasis on the performance of the second generations of hybrids.

Heredity (2007) 98, 268-273. doi:10.1038/sj.hdy.6800930; published online 14 February 2007

Keywords: genetic architecture; oviposition preference; epistasis; heterosis

\section{Introduction}

In herbivorous insects, changes in host plant preference for oviposition sites, feeding and mating are relevant to a wide range of evolutionary questions such as ecological specialization, host range formation and sympatric speciation (Jaenike and Holt, 1991; Via and Hawthorne, 2002; Fox et al., 2004 and reference therein). In addition, there is considerable interest in how evolutionary changes can impact herbivorous insects on agriculturally important plants, and how rapidly insects may adapt to utilizing unsuitable plant species.

Despite its special evolutionary importance, the genetic basis of the behavioural traits that influence choice of plant species for oviposition is relatively poorly understood. As with other behavioural traits (Meffert et al., 2002), heritabilities of oviposition preference within phytophagous insects populations are at the low-to-moderate level. In our previous study with the seed beetle Acanthoscelides obtectus heritability of oviposition preference was about 7.5\% (Tucić

Correspondence: Dr N Tucić, Department of Evolutionary Biology, Institute for Biological Research 'Siniša Stanković', Despota Stefana 142, 11060 Belgrade, Serbia.

E-mail: ntucic@ibiss.bg.ac.yu

Received 22 June 2006; revised 6 September 2006; accepted 23

November 2006; published online 14 February 2007 et al., 1997). This is within the range of oviposition preference heritability estimates for Drosophila buzzatti and D. aldrichi (Barker, 1992) and D. mojavensis (Lofdahl, 1987). There are three possible reasons for relatively low heritability of oviposition preference. First, it may reflect the past history of natural selection, in which case oviposition preference must be positively correlated with total fitness or its major components. As many attempts to understand the adaptive basis of host choice have been rather disappointing (see e.g. review in Meyhew (2001)), the cause of low heritabilities is still controversial. Secondly, environmental sources commonly reduce heritabilities, especially in the complex traits, such as oviposition preference. Finally, nonadditive genetic effects, such as dominance and epistasis, will reduce the heritability of a trait even in the presence of additive genetic variation (Merilla and Sheldon, 1999). Although nonadditive interactions have been treated in classical quantitative genetics theory as residual effects, several lines of evidence indicate that nonadditivity has important evolutionary implications (Lynch and Walsh, 1998; Merilla and Sheldon, 1999; Meffert et al., 2002). If, for instance, epistasis is the predominant component of genetic variance of an analysed trait, then crosses between populations can lead, depending on the relation between trait and fitness, to outbreeding depression or outbreeding enhancement in the first or in later generations. Also, empirical investigations have confirmed 
Fisher's (1958) prediction that long-term directional selection on a trait tends to select for dominance expression of genes in the direction of selection (Crnokrak and Roff, 1995; Sešlija and Tucić, 2003 and references therein). It is worth noting that the presence of high levels of dominance variance are consistent with the role of antagonistic pleiotropy in maintaining the genetic variance of fitnessrelated traits (Curtsinger et al., 1994; Roff, 1997).

Several studies have looked at the contribution of nonadditive genetic effects to phenotypic variation in oviposition preference in various insect species. However, the obtained results were inconsistent. For instance, Jaenike (1987), Keese (1996) and Craig et al. (2001) have detected dominance and/or epistasis, whereas the others (Sezer and Butlin, 1998; Tucić et al., 1999; Carroll et al., 2003; Fox et al., 2004) found that population differences in oviposition preference could be explained by complete additivity of genes. Accordingly, we still do not know how common or how necessary nonadditive genetic effects regarding oviposition behaviour are in the process of population divergence.

We herein investigate the genetic architecture of differences in oviposition preference between two populations of the seed beetle $A$. obtectus maintained in the laboratory for about ten years on different host seeds. One population was reared on the common bean ( $P$. vulgaris), the primary host of this beetle, while the other population is on a novel host, chickpea (C. arietinum). The first population was established from local populations collected from stored beans in the vicinity of Belgrade, an area where seed beetles were found exclusively on common bean seeds, and subsequently maintained in the laboratory on native host seeds. The second population was derived from the first and cultured in the laboratory on the new host, chickpea seeds. This latter population differs from the former, ancestral, population in several performance traits and in oviposition preference (Tucić et al., 1997). Thus, we examined the genetic architecture underlying the oviposition differences between two populations that have evolved in approximately 100 generations. We found it worthwhile to investigate these populations because there is a significant shortage of adequate empirical information concerning the genetic architecture of the phenotypic divergent traits in populations with known histories (but see Carroll et al., 2003 and references therein).

The goal of the present study is the elucidation of the relative contribution of additive genetic, nonadditive genetic, and maternal effects to the difference in oviposition preference between two populations evolved in the laboratory on the bean or chickpea seeds. To that end, we have conducted line-cross experiments in which we analysed the oviposition preference of purebred, hybrid and backcrossed individuals between two populations reared on both hosts. Such experimental design allowed us to examine whether genetic architecture depends on the host upon which insects are reared.

\section{Materials and methods}

\section{Life history of the bean beetle and experimental populations}

The bean beetle (A. obtectus; Coleoptera: Bruchidae) is a cosmopolitan pest of stored legumes. The primary host of this bruchid species is the common bean ( $P$. vulgaris). The larval survival, however, is satisfactory on chickpeas (C. arietinum) and other legumes.The females lay eggs in a cluster under or near a single seed. In contrast to other bruchids, which distribute their eggs in relation to host availability and previous egg density, there are no significant differences in the total number of eggs laid by $A$. obtectus females with different number of host seeds for oviposition (Parsons and Credland, 2003). Approximately four to six days later $\left(\right.$ at $\left.30^{\circ} \mathrm{C}\right)$, the eggs hatch and the first instar larvae burrow into the seed. Larval development and pupation are completed entirely within a single seed. Female adults mate within hours of emerging from the seed. Adults are facultatively aphagous, that is they need neither food nor water in the adult stage. $A$. obtectus has evolved to use dry seeds, most recently having evolved to use the storage environment, and is able to mate and complete reproduction using only metabolic water and resources acquired during larval development.

The first sample of the beetles used in this study was obtained from the base population maintained in the laboratory for about 10 years before the onset of the experiments. This population is synthesized by mass mating equal numbers of adults captured from three local populations $A$. obtectus the vicinity of Belgrade (Serbia) an area where the chickpea is not planted. The base population (henceforth the ' $\mathrm{P}$ ' population) is maintained at a large size (about 5000 individuals per generation) on $P$. vulgaris, c.v. 'gradistanac' seeds for about a 40-day interval.

The second population was derived from the base population. This population was established from a sample of about 1000 unsexed individuals randomly chosen from the base population and cultured on the new host, $C$. arietinum seeds. This population (henceforth the ' $C$ ' population) was subsequently maintained, at about 1000 adults per generation, on chickpea seeds for 102 generations before this experiment.

The experiments were conducted in a dark incubator at $30^{\circ} \mathrm{C}$ and relative humidity of about $70 \%$. All seeds bought in bulk from one source were frozen before use in the experimental treatments.

\section{Experimental design}

The $P$ and $C$ populations were crossed to produce $F_{1}$ and $F_{2}$ generations and two backcross generations, the $B_{1}$ and $B_{2}$ (i.e., $F_{1} \times C$ and $\left.F_{1} \times P\right)$. Reciprocals of all crosses were established, denoted as $\mathrm{rF}_{1}, \mathrm{rF}_{2}, \mathrm{rB}_{1}, \mathrm{rB}_{2}$. For each backcross, separate generations were raised in which the $\mathrm{F}_{1}$ parent differed reciprocally; e.g. $(\mathrm{C} \times \mathrm{P}) \times \mathrm{C}$ and $(\mathrm{P} \times \mathrm{C}) \times \mathrm{C})$. Thus, we created 14 distinct crosses. All crosses were set up with a minimum of 100 virgin individuals of each sex as parents.

In order to test the effects of rearing hosts on the genetic architecture of oviposition preference, we established two independent sets of crosses. The first set of crosses was set up in bottles containing bean seeds; for the second set of crosses the bottles contained chickpea seeds. Thus, within each set of crosses (i.e., from parental to $F_{2}$ generations) all larvae were raised from common beans, the host of the $\mathrm{P}$ population, or chickpea seeds, the host of the $C$ population, and then tested with regard to oviposition preference in a mixed-host environment. 
Assays of oviposition preference

Oviposition preference was measured in test Petri dishes (50-mm diameter) containing at their opposite ends an equal number (three) of the same sized bean and chickpea seeds. Within each cross type/rearing host single pair matings were performed between one-dayold females and males that had been randomly collected from the same cross type. As the presence or absence of a male through the lifetime of females had no effect on total fecundity (Parsons and Credland, 2003), these pairs were allowed to oviposit in separate test Petri dishes for $72 \mathrm{~h}$ (the oviposition rate of $A$. obtectus peaked $48-72 \mathrm{~h}$ after emergence). Female oviposition preference was defined as the proportion of eggs laid on chickpeas. In the parental populations and reciprocal crosses within $F_{2}$ generations oviposition preference was measured on about 200 females, while each reciprocal cross within $F_{1}$ and backcrosses consisted of about 100 females.

\section{Genetic analyses}

We tested for additivity, dominance, epistasis and maternal contribution to divergence of parental populations using joint scaling tests of phenotypic means, a weighted least squares multiple regression technique that scales for differences in population means (Lynch and Walsh, 1998, chapter 9). We used the expected phenotype of $\mathrm{F}_{2}$ offspring as the point of reference, that is its mean phenotype is presented as $m$, and all the composite effects of various types of gene action are defined to be zero in the $\mathrm{F}_{2}$ background.

Linear models of genetic architecture with up to nine composite genetic parameters were fitted to the 14 cross means. The composite genetic parameters were estimated by minimizing the sum of the weighted residual squares $\left(\mathrm{RSS}_{\mathrm{w}}\right)$. The weight is given as:

$$
\mathrm{RSS}_{\mathrm{w}}=\sum_{i=1}^{k} \frac{e_{i}^{2}}{S E_{i}^{2}}
$$

where $k$ is the number of crosses, $e_{i}^{2}$ is the difference between observed and predicted composite genetic effects and $S E_{i}^{2}$ are the standard errors of the estimated composite genetic effects. Under the assumption of normality, $\mathrm{RSS}_{\mathrm{w}}$ is $\chi^{2}$ distributed with the degrees of freedom equal to the number of lines minus the number of parameters in the model (Lynch and Walsh, 1998).

Following the suggestion of Bieri and Kawecki (2003), we used Akaike's information criterion (AIC) to select the model that contained the minimum number of parameters necessary to explain the observed generation means (which is referred to as the most parsimonious model). The model with the lowest AIC is the most parsimonious. Bieri and Kawecki (2003) showed that $\mathrm{AIC}=\mathrm{RSS}_{\mathrm{w}}+2 \mathrm{~K}+$ constant, in which $K$ is the number of parameters fitted in the model. The constant can be omitted because it plays no role in the ranking of the models.

The observed 14 generation means could be used to estimate the following parameters (notation follows Mather and Jinks (1982): an overall mean (m), additive $(d)$, dominance $(h)$, additive $\times$ additive epistasis (i), additive $\times$ dominance epistasis (j), dominance $\times$ dominance epistasis (1), an additive genetic maternal effect $\left(d_{\mathrm{m}}\right)$, a dominance genetic maternal effect $\left(h_{\mathrm{m}}\right)$, a cytoplasmic effect $(c)$ and a Y chromosome effect $(Y)$. To reduce the number of candidate models (there are $2^{9}=512$ possible models, $m$ being included in all models) we first tested models which included a cytoplasmic and $\mathrm{Y}$ - chromosome effects. In addition, following the suggestion of Bieri and Kawecki (2003), by pooling the three epistasis parameters $(i, j$ and $l)$ into $E$, and the two forms of maternal effects $\left(d_{\mathrm{m}}\right.$ and $\left.h_{\mathrm{m}}\right)$ into $\mathrm{M}$, we reduced the number of candidate models to $2^{4}=16$. Only if the most parsimonious models included $E$ or $M$, we expanded our models into all possible models including three forms of epistasis or the two forms of maternal effects. The significance of each parameter included in the most parsimonious model was tested with a likelihood-ratio test (Lynch and Walsh, 1998).

We used, following the suggestion of Fox et al. (2004), the Wald $\chi^{2}$ statistic to test the difference between the genetic parameters estimated from the two rearing hosts (m was not included in this analysis)

$$
\chi^{2}=\left(b_{1}-b_{2}\right)^{2} /\left[S E\left(b_{1}\right)^{2}+S E\left(b_{2}\right)^{2}\right]
$$

where $b_{1}$ and $b_{2}$ are the genetic parameters in each rearing host, and $S E\left(b_{1}\right)$ and $S E\left(b_{2}\right)$ are their s.e. The sum of the Wald $\chi^{2}$, with degrees of freedom equal to the number of parameters in the model, was used to assess the differences between models. In this analysis, we compared models with the same parametrization, that is for the purpose of hypothesis testing, both parameters were included in the models to be compared although only one was significantly different from zero.

\section{Results}

The extent of the difference in the mean oviposition preference for both parental populations and host seeds is shown in Figure 1. For both rearing hosts, $C$ females expressed about a $35 \%$ higher preference for chickpeas than $\mathrm{P}$ females, which was, as shown by two-way ANOVA (data were arcsin transformed), significant at the 0.001 level $\left(F_{1,571}=12.96\right)$. The effect of the rearing host was also highly significant $\left(F_{1,571}=20.2, P<0.001\right)$, indicating that females tend to prefer to oviposit their eggs on the seeds they have already experienced. However, there was no population $\times$ rearing host interaction $\left(F_{1,571}=1.18, P>0.05\right)$.

Figure 2 shows the oviposition preference means of the 14 crosses and estimates of the composite genetic effects are summarized in Table 1 . The reciprocal crosses are

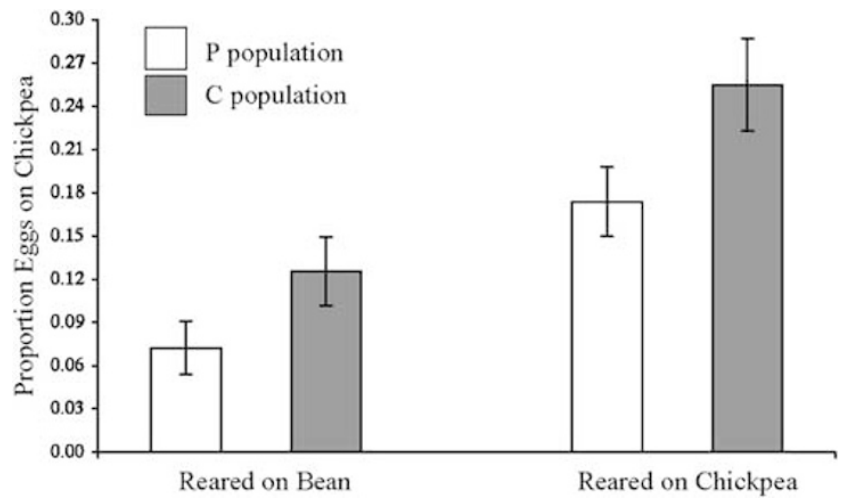

Figure 1 The mean oviposition preference ( \pm s.e.) of $\mathrm{P}$ and $\mathrm{C}$ beetles reared from bean and chickpea seeds. 


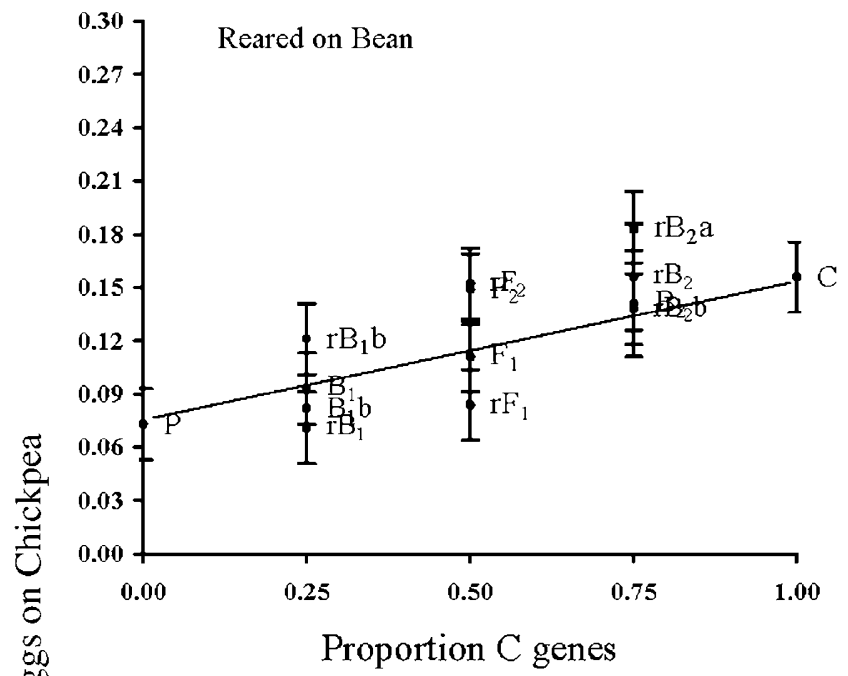

Table 1 Estimates of composite genetic effects underlying differgence in oviposition preference between $\mathrm{P}$ and $\mathrm{C}$ populations

\begin{tabular}{lcc}
\hline Parameter & Reared on bean & Reared on chickpea \\
\hline$m$ & $0.1526 \pm 0.0147$ & $0.3698 \pm 0.0658$ \\
$d$ & - & $-0.0516 \pm 0.0129$ \\
$h$ & - & $-0.3632 \pm 0.1588$ \\
$i$ & $-0.0536 \pm 0.0280$ & $-0.1526 \pm 0.0629$ \\
$j$ & $-0.1344 \pm 0.0311$ & - \\
$l$ & $-0.0579 \pm 0.0268$ & $0.1562 \pm 0.0765$ \\
$\chi^{2}$ & $8.85 \mathrm{NS}$ & $4.59 \mathrm{NS}$ \\
\hline
\end{tabular}

Abbrevaition: NS, not significant.

Estimates represent the composite genetic effects from the Akaike's information criterion (AIC) most parsimonious model. Model parameters: $m$, overall mean; $d$, additive; $h$, dominance $i$, additive-additive epistasis; $j$, additive-dominance epistasis; $l$, dominance-dominance epistasis. $\chi^{2}$, goodness of fit of the model to the real data.

that overall models did not differ significantly between rearing hosts or that the rearing host affected any of the individual parameters.

According to Mather and Jinks (1982), under purely additive gene action, $F_{1}$ and $F_{2}$ hybrids have an expected trait value equal to the average of both parental values, whereas under dominant gene action (without epistasis), $\mathrm{F}_{2}$ lies between $\mathrm{F}_{1}$ and the midparent. However, as can be seen in Figure 2, there is a tendency that the $F_{2}$ hybrids, reared from both host seeds, have a higher trait value than assumed under additive and/or dominant gene action. As shown by Gilchrist and Partridge (1999), higher or lower than expected $F_{2}$ hybrid values can be caused by epistatic interactions and/or maternal effects. In the absence of significant maternal effects, we were able to test the contribution of epistasis to $F_{2}$ performance by using the $C^{\prime}$ scaling test (Bieri and Kawecki, 2003): $C^{\prime}=-2[i]-[l]$, where $i$ is the additive $\times$ additive and $l$ is dominance $\times$ dominance epistasis (the standard error of $C^{\prime}$ are estimated from the sampling variancecovariance matrix of the parameter estimates). A significantly positive $C^{\prime}$ indicates hybrid enhancement due to epistatic effects, whereas a significant negative $C^{\prime}$ indicates hybrid breakdown. Using our data, large positive values of $C^{\prime}$ were found for ovipostion preference of females reared from beans $\left(C^{\prime}=16.51 \pm 6.58\right.$, $P<0.01)$ as well as for females reared from chickpeas $\left(C^{\prime}=14.90 \pm 7.09, P<0.05\right)$.

the oviposition preference of the females reared on bc rearing hosts.

The model including additive genetic effects (d), dominance $(h)$ and additive $\times$ additive (i) and dominance $\times$ dominance epistasis (l) was the AIC most parsimonious when females were reared from chickpeas $\left(\mathrm{RSS}_{\mathrm{w}}=4.59, \mathrm{df}=9,0.75<P<0.90\right)$. Although the two epistasis parameters were included in most parsimonious model, we removed them from the model to assess if they significantly improve the fit of the model (see Bieri and Kawecki (2003) for testing epistasis). The likelihoodratio test $\Lambda=11.60$, which follows $\chi^{2}$ distribution with $\mathrm{df}=2(P<0.01)$, showed that $i$ and 1 parameters indeed increase the fit of the model. The most parsimonious model for the females reared from beans excludes additive and dominance effects, but includes $i, j$ and $l$ epistatic effects, with all epistasis parameters being significant $\left(\mathrm{RSS}_{\mathrm{w}}=8.85, \mathrm{df}=10,0.50<P<0.75\right)$. However, the Wald $\chi^{2}$ test $\left(\chi^{2}=0.82, \mathrm{df}=5, P>0.05\right)$ indicated

\section{Discussion}

The results presented here suggest that preference for the chickpea was stronger in the population evolved for 102 generations in the laboratory on the chickpea, rather than in the population maintained on the common bean (Figure 1). This finding is consistent with our previous results, which showed a divergence of host preference between the same ancestral and derived populations (Tucić et al., 1995, 1997). Our studies of A. obtectus accord with those of Calosobruchus maculatus (Wasserman and Futuyma, 1981; Credland, 1987; Fox, 1993; Messina, 2004; but see Kawecki and Mery, 2003 for contrasting results) in demonstrating a divergence of host preference between populations maintained on different host species. 
The present study reveals complex genetic architecture underlying population differentiation with respect to oviposition preference of $A$. obtectus. Our findings are in accordance with those of several insect species in demonstrating substantial nonadditive genetic effects of population differences in oviposition behaviour (Fox et al., 2004 and reference therein). Moreover, in summary of a literature search on nonadditive genetic effects in animal behaviour, Meffert et al. (2002) found that about $64 \%$ of studies indicated at least one nonadditive effect (37\% of the records showed two or more nonadditive effects of genes) and that dominance was present in $40 \%$ of the behavioural traits examined. Hence, our experimental results are not peculiar in exhibiting the strong influences of nonadditive genetic effects.

As the two populations of $A$. obtectus examined here have evolved on different host species, that is they experienced host-specific selection, it was necessary to assess the genetic architecture of oviposition preference on both of the host in which females developed. Although there was no evidence that the AIC most parsimonious models differed between rearing hosts, the host from which females were reared affected whether additive and dominance effects were detectable, and which types of epistasis influenced line means (Table 1). Statistically detectable additive and dominance genetic effects were present in the AIC most parsimonious model only when females were reared on the chickpea. This make sense because, according to Hoffmann and Parsons (1991), the additive genetic variance of fitnessrelated traits might be expected to increase under conditions (i.e. host seeds) that are rarely experienced in nature. Also, the detectable negative dominance effect on the chickpea is not unexpected because the directional selection on a trait, as pointed out by Fisher (1958), tends to select for the dominance expression of genes in the direction of selection.

Finally, the results reported here have consequences for the ongoing discussion regarding the genetic mechanisms underlying heterosis and outbreeding depression. The most parsimonious models on both rearing hosts consistently suggested a contribution of negative additive $\times$ additive epistasis to the divergence of oviposition preference between the $\mathrm{P}$ and $\mathrm{C}$ populations. This indicates a positive effect of epistasis in the performance of the second-generation hybrids, as evidenced in the significantly positive values of $C^{\prime}$ (see results). This effect is more pronounced when the rearing host was the bean, because there both additive $\times$ additive and dominance $\times$ dominance epistasis contributed to enhancement of the $F_{2}$ hybrids. Several previous studies have also demonstrated a positive contribution of epistasis to fitness-related traits in second-generation hybrids (Blows et al., 1995; Fenster and Galloway, 2000; Bieri and Kawecki, 2003).

As can be seen in Figure 2, there is a tendency that the $F_{1}$ values are lower, and the $F_{2}$ values higher, than the midparent. Although quite unusual, such results are theoretically predicted in the model of heterosis developed by Schnell and Cockerham (1992). According to this model, heterosis with completely multiplicative action between genes can be negative in the $F_{1}$ and positive in the $F_{2}$ only if the highest frequency of alleles at two loci that increase the trait values is found in different parent populations. This model mainly concerns the complex quantitative traits, which are products of several subtraits, each controlled by a different set of genes. The oviposition preference is a notoriously complex trait, which includes, for example, the sensory organs involved in the oviposition choices, and the central processing of inputs from different organs and receptors. Hence, we can think that the Schnell-Cockerham model might account for the observed pattern of oviposition preference of $A$. obtectus in the $\mathrm{F}_{1}$ and $\mathrm{F}_{2}$ hybrids.

\section{Acknowledgements}

We thank C Fox and three anonymous referees for helpful comments and suggestions. This study was supported by the Ministry for Science, Technology and Development of Serbia, project No. 143033.

\section{References}

Barker JSF (1992). Genetic variation in cactophilic Drosophila for oviposition on natural substrates. Evolution 46: 1070-1083.

Bieri J, Kawecki TJ (2003). Genetic architecture of differences between populations of cowpea weevil (Callosobruchus maculatus) evolved in the same environment. Evolution 57: 274-287.

Blows MW, Bakker K, Sokolowski MB (1995). The expression of additive and nonadditive genetic variation under stress. Genetics 140: 1149-1159.

Carroll SP, Dingle HG, Famula TR (2003). Rapid appearance of epistasis during adaptive divergence following colonization. Proc Royal Soc B 270: S80-S83.

Craig TP, Horner JD, Itami JK (2001). Genetics, experience, and host plant preference in Eurosta solidaginis: implications for host shifts and speciation. Evolution 55: 773-782.

Credland PF (1987). Effects of host change on the fecundity and development of an unusual strain of Callosobruchus maculatus (F.) (Coleoptera: Bruchidae). J Stor Prod Res 23: 91-98.

Crnokrak P, Roff DA (1995). Dominance variance: associations with selection and fitness. Heredity 75: 530-540.

Curtsinger JW, Service PM, Prout T (1994). Antagonistic pleiotropy, reversal of dominance, and genetic polimorphism. Am Nat 144: 210-228.

Fenster CB, Galloway LF (2000). The contribution of epistasis to the evolution of natural populations: a case study of an annual plant. In: Wolf JB, Brodie ED, Wade MJ (eds.) Epistasis and the Evolutionary Process. Oxford University Press: New York. pp. 232-244.

Fisher RA (1958). The Genetical Theory of Natural Selection, 2nd edn. Dover Publications: New York.

Fox CW (1993). A quantitative genetic analysis of oviposition preference and larval performance on two hosts in the bruchid beetle, Callosobruchus maculatus. Evolution 47: 166-175.

Fox CW, Stillwell RC, Amarillo-S AR, Szesak ME, Messina FJ (2004). Genetic architecture of population differences in oviposition behaviour of the seed beetle Callosobruchus maculatus. J Evol Biol 17: 1141-1151.

Gilchrist AS, Partridge L (1999). A comparison of the genetic basis of wing size divergence in three parallel body size clines of Drosophila melanogaster. Genetics 153: 17751787.

Hoffmann AA, Parsons PA (1991). Evolutionary Genetics and Environmental Stress. Oxford University Press: Oxford.

Jaenike J (1987). Genetics of oviposition-site preference in Drosophila tripunctata. Heredity 59: 363-369.

Jaenike J, Holt RD (1991). Genetic variation for habitat preference: evidence and explanation. Am Nat 137: S67-S90.

Kawecki TJ, Mery F (2003). Evolutionary conservation of geographic variation in host preference in Callosobruchus maculatus. Ecol Ent 28: 449-456. 
Keese MC (1996). Feeding responses of hybrids and the inheritance of host-use traits in leaf feeding beetles (Coleoptera: Chrysomelidae). Heredity 76: 36-42.

Lofdahl K (1987). A genetic analisis of habitat selection in the cactophilic species, Drosophila mojavensis. In: Huettel MD (ed) Evolutionary Genetics of Invertebrate Behaviou. Plenum Press: New York. pp 153-163.

Lynch M, Walsh B. (1998). Genetics and Analysis of Quantitative Traits. Sinauer Associates, Inc.: Sunderland.

Mather K, Jinks JL (1982). Biometrical Genetics; the Study of Continuous Variation. Chapman and Hall: London.

Meffert LM, Hicks SK, Regan JL (2002). Nonadditive genetic effects in animal behavior. Am Nat 160: S198-S213.

Merilla J, Sheldon BC (1999). Genetic architecture of fitness and nonfitness traits; empirical patterns and development of ideas. Heredity 83: 103-109.

Messina FJ (2004). How labile are the egg-laying preference of seed beetles? Ecol Ent 29: 318-326.

Meyhew PJ (2001). Herbivore host choice and optimal bad motherhood. Trends Ecol Evol 16: 165-167.

Parsons DMJ, Credland PF (2003). Determinants of oviposition in Acanthoscelides obtectus: a nonconformist bruchid. Physiol Ent 28: 221-231.

Roff DA (1997). Evolutionary Quantitative Genetics. Chapman and Hall: New York.

Schnell FW, Cockerham CC (1992). Multiplicative vs arbitrary gene action in heterosis. Genetics 131: 461-469.
Šešlija D, Tucić N (2003). Selection for developmental time in bean weevil (Acanthoscelides obtectus) correlated response for other life history traits and genetic architecture of line differentiation. Ent Expt Appl 106: $19-35$.

Sezer M, Butlin RK (1998). The genetic basis of oviposition preference differences between sympatric host races of the brown planthopper (Nilaparvata lugens). Proc Royal Soc B 265: 2399-2405.

Tucić N, Milanović D, Mikuljanac S (1995). Laboratory evolution of host plant utilization in the bean weevil (Acanthoscelides obtectus). Genet Sel, Evol 27: 491-502.

Tucić N, Mikuljanac S, Stojković O (1997). Genetic variation and covariation among life history traits in populations of Acanthoscelides obtectus maintained on different hosts. Ent Exp Appl 85: 247-256.

Tucić N, Milanović D, Gliksman I, Šešlija D (1999). Genetic basis of oviposition preference in bean weevil (Acanthoscelides obtectus). Arch Biol Sci 51: 89-98.

Via S, Hawthorne DJ (2002). The genetic arichitecture of ecological specialization: correlated gene effects on host use and habitat choice in pea aphids. Am Nat 159: S76-S88.

Wasserman SS, Futuyma DJ (1981). Evolution of host plant utilization in laboratory populations of the southern cowpea weevil, Callosobruchus maculatus Fabricius (Coleoptera: Bruchidae). Evolution 35: 605-617. 